\title{
Numerical simulation of vortex sheet evolution
}

\author{
Robert KRASNY * \\ Courant Institute of Mathematical Sciences, New York University, 251 Mercer St., New York, NY 10012, USA
}

\begin{abstract}
Recent research on the numerical simulation of vortex sheet evolution is discussed. Applications are presented to a periodic vortex sheet and to the vortex sheet shed behind a finite-span wing.
\end{abstract}

\section{Introduction}

This article discusses recent research on the vortex sheet model of a free shear layer. Diffusion of vorticity is neglected in this model and the shear layer is approximated by a sharp interface along which the fluid is allowed to slip. The vortex sheet evolution equation is strongly nonlinear and it is difficult to solve numerically. Before discussing the numerical issues, it is important to note the problems' mathematical context.

\section{Analytical results for vortex sheet evolution}

Consider an infinite flat vortex sheet of constant strength. A classical result is that this vortex sheet is linearly unstable, with the perturbation growth rate proportional to the perturbation wavenumber. This phenomenon is known as "Kelvin-Helmholtz instability" in the fluid dynamics literature and "ill-posedness in the sense of Hadamard" in the mathematical literature. Birkhoff (1962) conjectured that the nonlinear problem for analytic perturbations is well posed but that this analyticity might be lost at a finite critical time $t_{\mathrm{c}}$.

Recent analytical work confirms these conjectures. Analytical vortex sheets exist locally in time (Sulem, Sulem, Bardos and Frisch 1982). Moore (1984, 1979) presented an asymptotic analysis indicating that a singularity can form in the shape of an evolving periodic vortex sheet. At the critical time, the vortex sheet remains continuously differentiable but has an infinite jump discontinuity in the curvature at isolated points. Meiron, Baker and Orszag (1982) obtained good agreement with this result, using Taylor series in time. Caflisch (1987) describes work concerning the rigorous validity of Moore's asymptotics.

Less is rigorously known about the finite-span wing problem because tip singularities are present in the initial data and the local existence theory doesn't apply. Early work by Kaden (see Saffman and Baker 1979) suggested that the sheet's tips roll up instantaneously into spirals. Pullin and Phillips (1981) have studied self-similar solutions in which single and double branched spirals form instantaneously in flat vortex sheets possessing certain types of singularities. This suggests that the formation of such a singularity in a time dependent problem causes the vortex sheet to roll up into a spiral.

* Present address: Mathematics Department, University of Michigan, Ann Arbor, MI 48109. 


\section{Numerical difficulties}

Two of the vortex sheet's analytical properties mentioned above lead to computational difficulties. These properties are 1) short wavelength instability and 2) singularity formation and spiral roll-up.

Short wavelength instability of the governing equation implies that a consistent discrete approximation will be unstable. In this situation, refining the mesh may not improve the computation's accuracy. This is because computer roundoff error introduces spurious perturbations which grow in amplitude. For the periodic problem, accurate numerical solutions can be obtaincd up to the vortex sheet's critical time $t_{\mathrm{c}}$, either by using higher machine precision or by filtering out the roundoff error in Fourier space (Krasny 1986a). In particular, Rosenhead's point vortex approximation converges up to $t_{\mathrm{c}}$ and the numerical results support the picture of singularity formation obtained by Moore and Meiron, Baker and Orszag.

One consequence of the loss of smoothness due to singularity formation is that a higher order discretization can become inconsistent. Furthermore, the discretization should be capable of approximating a spiral curve shape.

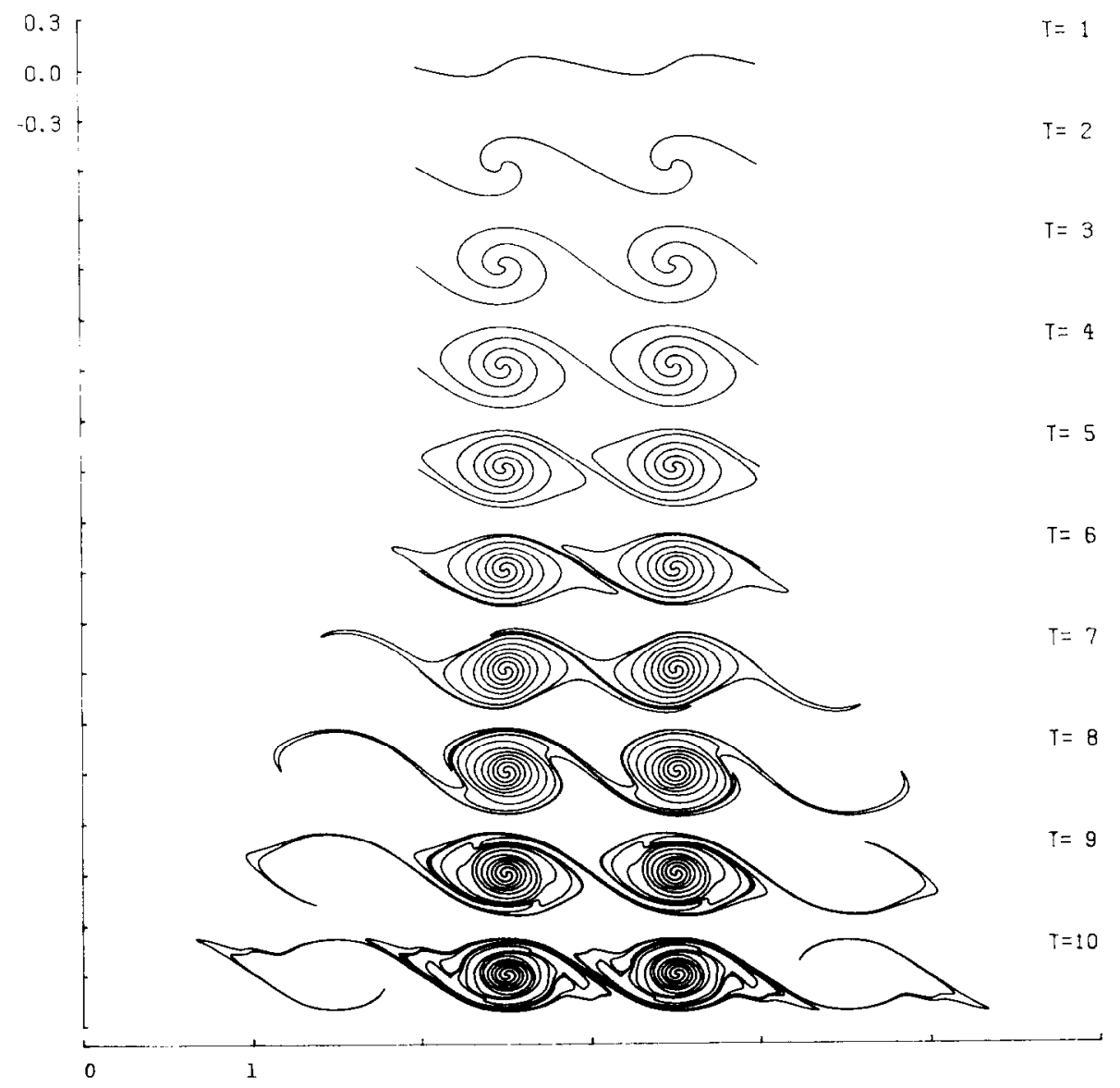

Fig. 1. A periodically perturbed vortex sheet. The initial perturbation was an eigenfunction of the linearized equation. The plotted curve represents two periods in the circulation parameter. The smoothing parameter, analogous to a finite core size, had the value 0.5 , based upon unit wavelength. The sheet rolls up into a double-branched spiral whose outer turns are elliptically deformed as time progresses. At late times, the outer turns are captured by the vortices in adjacent periods. 


\section{Desingularization}

An approach which diminishes these difficulties is based upon the vortex blob method of Chorin and Bernard (1973). An artificial smoothing parameter $\delta$ is introduced into the Cauchy principal value integral which defines the vortex sheet's velocity. For example, in the finite-span problem, the singular Cauchy kernel $K(z)=1 / 2 \pi \mathrm{i} z$ is replaced by the smooth approximate kernel $K_{\delta}(z)=K(z)|\mathrm{z}|^{2} /\left(|z|^{2}+\delta^{2}\right)$.

The smoothed equation has a numerically tractable solution for the following reasons (Krasny 1986b). First, linear stability analysis shows that the smoothing reduces the perturbation growth rates. This means that the computer precision requirement is less severe than for the unsmoothed equation. Second, singularity formation does not occur and therefore a higher order spatial discretization can attain its theoretical convergence rate. Solutions of the smoothed equation have a finite number of turns and can be well approximated using a reasonable number of mesh points. Some solutions of the desingularized equations are shown in fig. 1-3. In these results, the time and spatial discretization errors are negligible.

If the smoothed solutions converge as the smoothing parameter vanishes, then the limit function is a possible weak solution to the vortex sheet evolution equation. The standard convergence theory for vortex methods (see Anderson and Greengard 1985) doesn't apply here but DiPerna and Majda (1987) have begun studying convergence questions for vortex sheets. Numerical evidence indicates that as the smoothing parameter vanishes, the smoothed solutions

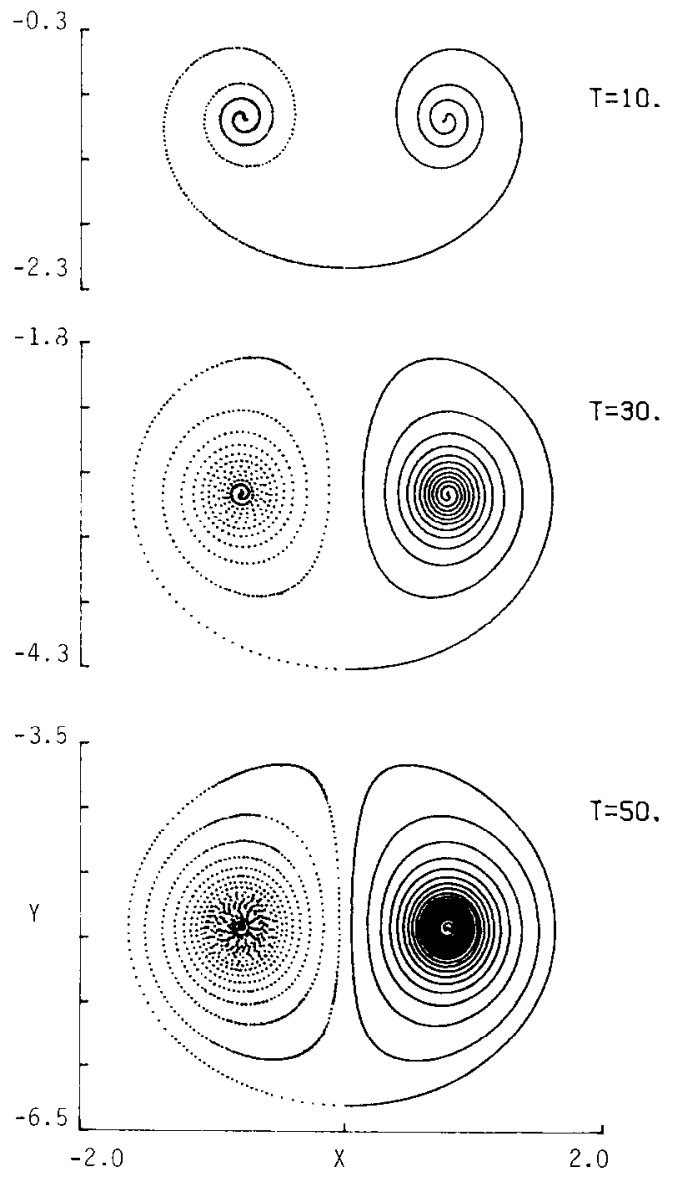

Fig. 2. The vortex sheet shed by an elliptically loaded wing. The vortex blob positions are plotted on the left and an interpolating curve is plotted on the right. The value of the smoothing parameter is 0.2 based upon unit half-span. The sheet rolls up into counterrotating vortices which propagate downward. At late times, the vortices are elliptically deformed. 


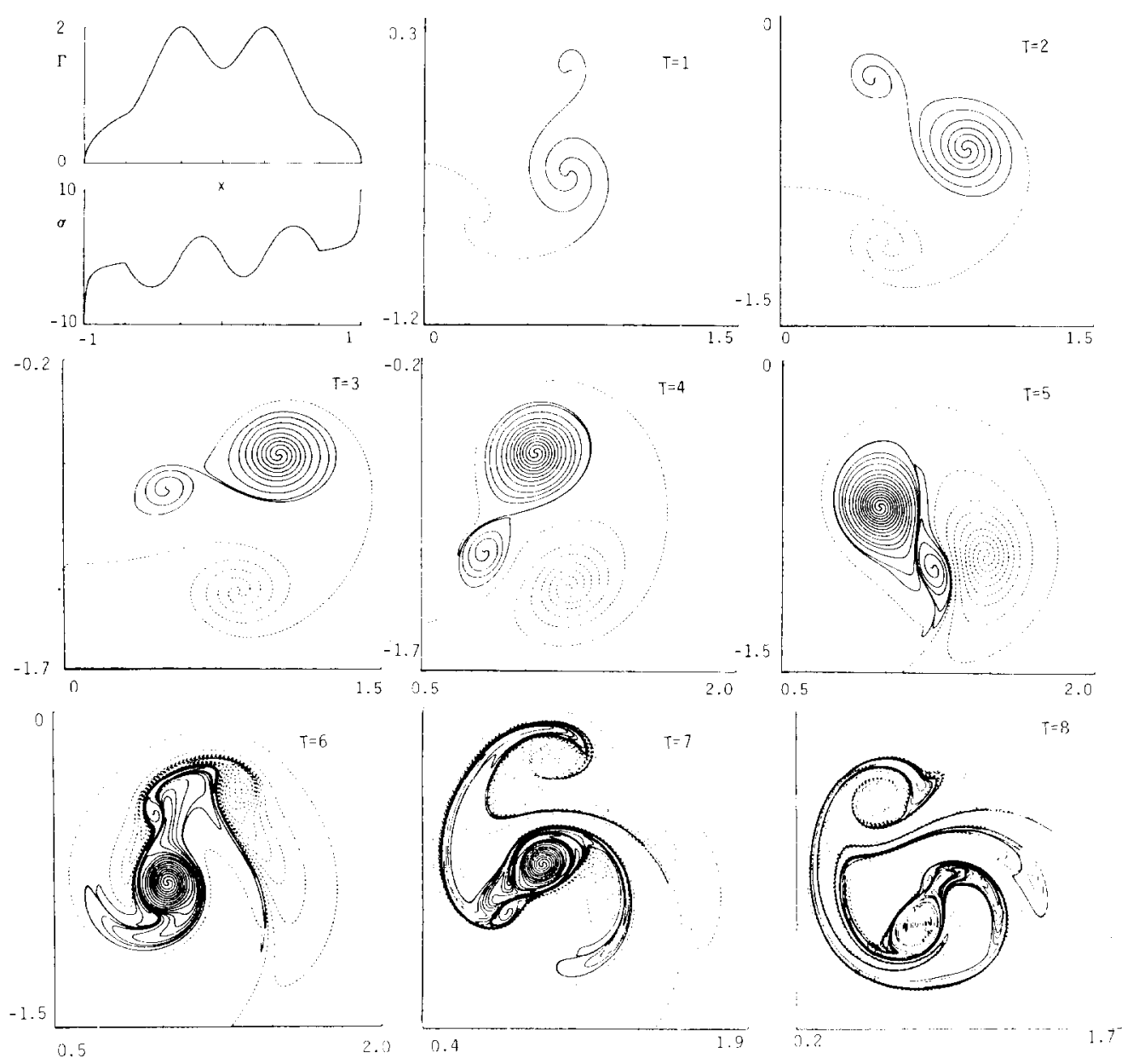

Fig. 3. The vortex sheet due to a simulated fuselage-flap loading on a finite-span wing. The circulation and initial vortex sheet strength are plotted across the span in the upper left frame. The right half-span region of the wake is plotted in the remaining frames. The solid and dotted portions of the vortex sheet signify regions of oppositely signed vortex sheet strength. The value of the smoothing parameter is 0.1 based upon unit half-span. The two liked-signed vortices rotate around one another and the small tip vortex gradually merges with the larger flap vortex. The structure also propagates as a dipole, due to the presence of oppositely signed vorticity within each half-span region. At $t=5$ the tip vortex is colliding with the fuselage vortex. At later times the outer turns of each vortex are stripped away from the cores. Portions of these outer turns becomes concentrated into long sweeping fronts which are no longer associated with a particular core.

coverage to a spiral past the vortex sheet's singularity formation time (Krasny 1986b, 1987). Other candidate weak solutions for the vortex sheet equation are being studied. Baker and Shelley (1987) study the limit of vanishing thickness for a periodic layer of constant vorticity. Tryggvason (1987) has shown that vortex-in-cell calculations have an inherent smoothing property which resembles that of the vortex blob method. Uniqueness for weak solutions past the vortex sheet's critical time has not yet been resolved.

\section{Conclusions}

Several general guidelines for vortex sheet computation can be drawn from this research. Singularity formation in an analytic vortex sheet can be studied using the point vortex 
approximation, if computer roundoff error is controlled. However, some kind of smoothing is necessary to follow the vortex sheet's evolution past the critical time. It is hoped that this methodology can be extended to include physical mechanisms that are neglected in the present model.

\section{Acknowledgements}

This work has been supported by the National Science Foundation, the Air Force Office of Scientific Research URI grant \#86-0352 and the NASA-Ames Research Center grant NAG $2-345$.

\section{References}

Anderson, C. and Greengard, C. (1985) On vortex methods, SIAM J. Numer. Anal. 22, 413.

Baker, G.R. and Shelley, M. (1987) On the relation between thin vortex layers and vortex sheets, in preparation.

Birkhoff, G. (1962) Helmholtz and Taylor instability, Proc. Symp. Appl. Math. XIII A.M.S. 55.

Caflisch, R.E. (1987) Nonlinear analysis for the evolution of vortex sheets, IUTAM '87, Fluid Dyn. Res. 3 (1988) $75-77$.

Chorin, A.J. and Bernard, P.S. (1973) Discretization of a vortex sheet, with an example of roll-up, J. Comput. Phys. 13, 423.

DiPerna, R.J. and Majda, A. (1987) Concentrations in regularizations for 2-D incompressible flow, Commun. Math. Phys. $108,667$.

Krasny, R. (1986a) A study of singularity formation in a vortex sheet by the point vortex approximation, J. Fluid Mech. 167, 65 .

Krasny, R. (1986b) Desingularization of periodic vortex sheet roll-up, J. Comp. Phys. 65, 292.

Krasny, R. (1987) Computation of vortex sheet roll-up in the Trefftz plane, J. Fluid Mech., 184, 123.

Meiron, D.I. Baker, G.R. and Orszag, S.A. (1982) Analytic structure of vortex sheet dynamics, 1. Kelvin-Helmholtz instability, J. Fluid Mech. 114, 283.

Moore, D.W. (1979) The spontaneous appearance of a singularity in the shape of an evolving vortex sheet, Proc. Roy. Soc. London A 365, 105.

Moore, D.W. (1984) Numerical and analytical aspects of Helmholtz instability. Proc. XVI IUTAM Conf., eds. Nordson and Olhoff (North-Holland, Amsterdam) 263.

Pullin, D.I. and Phillips, W.R.C. (1981) On a generalization of Kaden's problem. J. Fluid Mech. 104, 45.

Saffman, P.G. and Baker, G.R. (1979) Vortex interactions, Ann. Rev. Fluid Mech. 11, 95.

Sulem, C., Sulem, P.L., Bardos, C. and Frisch, U. (1981) Finite time analyticity for the two and three dimensional Kelvin-Helmholtz instability, Comm. Math. Phys. 80, 485.

Tryggvason, G. (1987) Simulation of vortex sheet roll-up by vortex methods, to appear. J. Comp. Phys. 